\title{
Exploring the Translation Mode for Scientific Discourses Under the Framework of Information Dualism
}

\author{
Xiaofeng Lei \\ School of Foreign Languages, Northwestern Polytechnical University, Xi’an, China \\ leixiaofeng7425@163.com
}

\begin{abstract}
This paper aims to offer scientists and engineers some support in their translation of scientific discourse. As a practical branch of English, scientific English has always played important roles in the development of all countries, such as exchanging technological information, accelerating scientific communications, broadening and deepening (bi/) multilateral cooperation across the world. For various reasons, however, problems with the translation of scientific discourse exist in large numbers, which is not conducive to the input of advanced western science and technology into China or the other way. Within the framework of information dualism, namely, "information equivalency" and "information transitivity", combining the linguistic and extra-linguistic factors, the author of this paper has designed a practical, easyto-handle translation mode for scientific discourse, which should prove to be of great help to scientists with respect to acquiring scientific information, and, accordingly, improving their work efficiency.
\end{abstract}

Keywords- information dualism, scientific English, translation mode

\section{INTRODUCTION}

Scientific English is closely related to our life and work. Along with the rapid development of science and technology and ever-increasing international communication and cooperation, English is becoming more and more important for science and technology. Due to the fact that the research on translation from Chinese into scientific English is far from adequate and that the level of expertise of the personnel involved varies, the quality of translation products is often more or less unsatisfactory [2]. Researchers usually adopt literary translation theory to conduct translation activities, which results in an abundance of problems. However, colleges and universities are important fronts of research work, where scientists need to read a large volume of literature (mainly in English) to acquire advanced scientific knowledge and learn about present development trends in the area of technological innovation. However, constrained by limited theoretical knowledge of English, lots of scientific researchers in China's colleges and universities have difficulty in comprehending scientific and technical texts.

\section{DISCUSSED PROBLEMS}

In view of the problems mentioned above, the author has designed the practical translation mode for scientific discourse, by combining Informatics theory and the features of scientific English texts. This mode has the explicit goal of maintaining "information equivalency" and "information transitivity", the two important criteria put forward by Chinese scholar Feng Zhijie in 1996[1] [3]. This translation mode starts with the thorough analysis of the source text in terms of linguistic factors (syntax, semantics and discourse) and extra-linguistic factors (the author's intention, cognitive perspective, cultural aspects, etc.), the application of scientific and practical translation methods, and finally, the reproduction of the "equivalent" information.

This can be simply illustrated as follows:

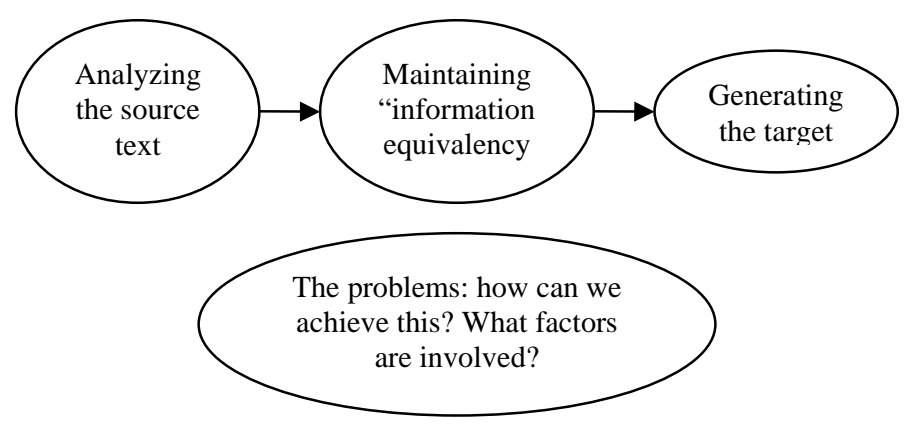

Figure 1: the problems to be discussed

\section{THEORETICAL BACKGROUND: INFORMATION DUALISM}

Informatics is an academic field or discipline that studies the measurement, transfer and transformation rules of information. It is mainly concerned with the common laws of ubiquitous information transfer in communication and control systems, and the basic theories of issues such as the best way to approach, measure, transfer and store information [4]. From the perspective of information theory, the essence of translation is in information conversion, transferring the original information through a translator to the target language. Accordingly, the general requirement of translation is to transfer the source information as completely as possible to target readers.

Under the framework of informatics, the inclusion degree of the source information in the target text is termed "Information Equivalency", while the degree that the target readers get access to the original information through the target text is termed information transitivity of the target text, "Information Transitivity" for short. In the present paper, information dualism refers to information 
equivalency and information transitivity as applicable to the translation of the scientific discourse [1].

Scientific English is a practical branch of English. Its language is characterized by the extensive use of nominalization, wide use of the passive voice, compact sentence structuring, and fixed sentence patterns. In addition, the translation of scientific discourses is targeted mainly at scientific workers or readers who occasionally need a particular item of scientific or technological information. They read scientific materials mainly to be able to quickly access accurate information. Therefore, the goal all translators must be to strive to maintain information equivalency and information transitivity of the source text in the target one.

The Analysis of the Source Text- Information Equivalency (the basis)

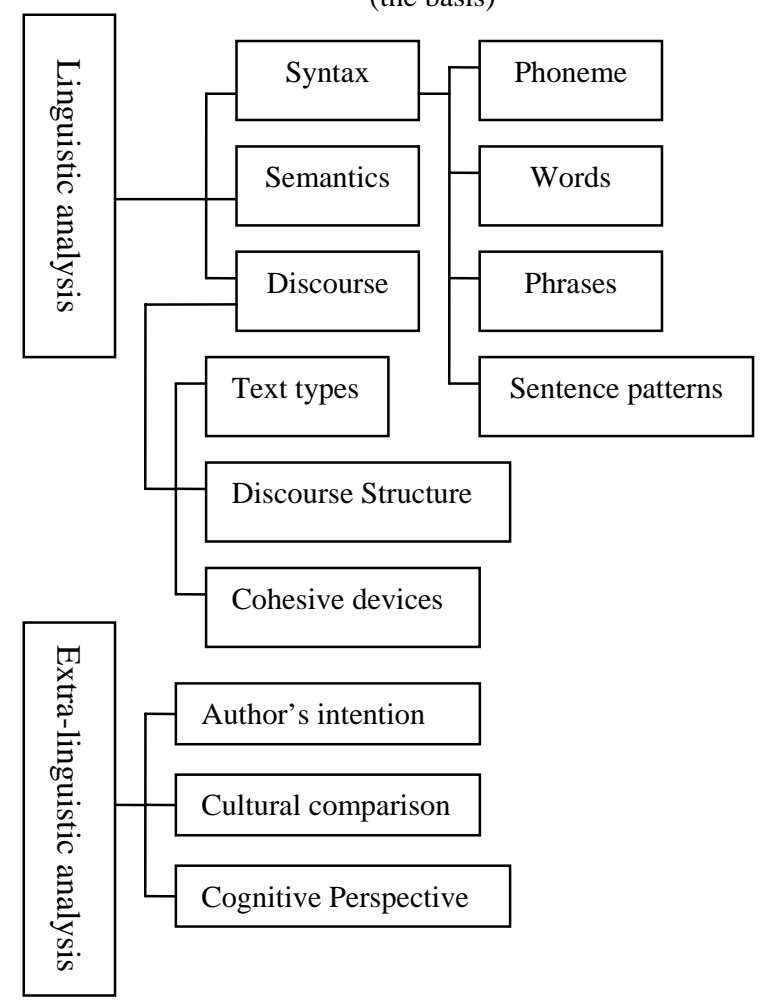

The Generation of the Target Text- Information Transitivity (the effect)

Note: Top-down $=$ top-down translation mode (starting from the treatment of textual factors to the handling of phonemes); inversion (translating from the back to the front); cutting (for convenience, cutting a whole part into different parts); embedding (integrating a separate part into another, normally a larger unit); Compre. $=$ comprehensive method (using more than one method at the same time).

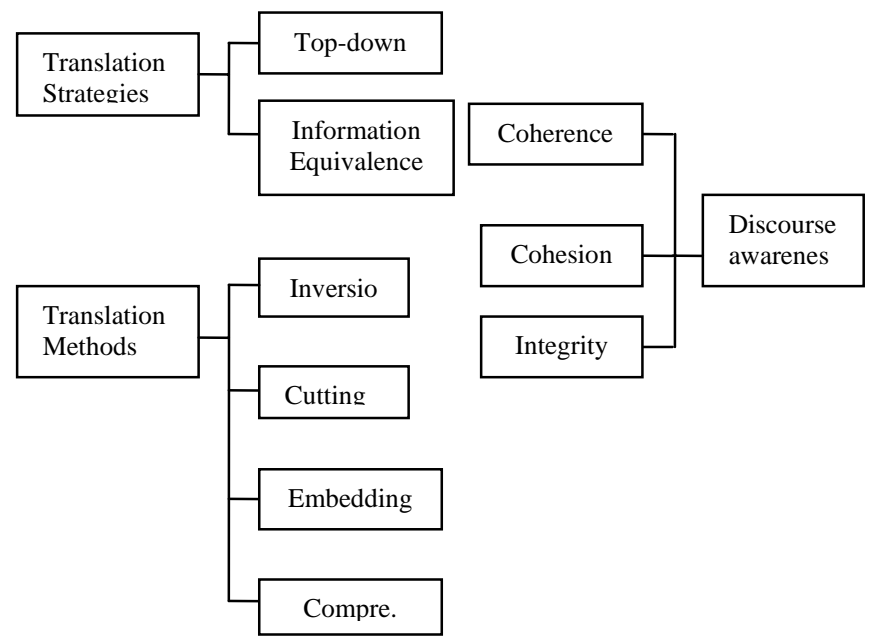

Figure 2: the translation mode for scientific discourses under the framework of information dualism

\section{TRANSLATION MODE FOR SCIENTIFIC DISCOURSE} UNDER THE FRAMEWORK OF INFORMATION DUALISM

As figure 1 shows, under the framework of information dualism, the translation of scientific discourse involves 2 phases: the analysis of the source text and the generation the target text.

Phase 1: the analysis of the source text

Here, translators, in order to fully understand the original text, need to make a thorough analysis of the linguistic factors (syntax, semantics, discourse, etc.) and extra-linguistic factors (the author's intention, bilingual cultural similarities and differences, bilingual readers' cognitive psychology, etc.) of the source text. Then, through the bottom-up mode of information processing (starting from phonemes, then words, sentences, to discourse), translators can eventually figure out the textual as well as the pragmatic meanings of the source text, which lays a solid foundation for ensuring information equivalency in the rendering process.

Phase 2: the genen of the target text

At this point, translators, being aware of discourse conventions - discourse integrity, textual cohesion and coherence - adopt translation methods such as inversion, cutting, embedding and comprehensive methods, through the "top-down" translation mode (starting processing from discourse, followed by sentences, phrases, words, and phonemes), and by maintaining information equivalence (equivalency) and information acceptability (transitivity). The final product should be a satisfactory target text.

\section{EXAMPLES AND ANALYSES}

In order to illustrate the feasibility and effectiveness of this translation mode, I cite two paragraphs from different sources, to exemplify how this translation mode works with it. Because it is probably not possible to illustrate all the steps involved in translation in one simple example, only a 
few crucial translation techniques are only demonstrated here.

\section{A. Example 1[5]}

(1) The beneficial impacts of infrastructure on women can be profound, often extending beyond the commonly cited impacts of water and sanitation infrastructure on household health or women's time allocation. (2)But ensuring such outcome requires foresight and attention to detail during project planning.

Analyses:

This is actually the English version of one Chinese paragraph. Compared with English, Chinese seems sometimes loose and less concise, of which the translator is quite aware. After a thorough analysis of the syntax, semantics and discourse of that short Chinese paragraph, the translator clearly has these points in mind: 1) "women" is topic of this paragraph, thus must serve as the center of attention to readers; 2) two sentences in the source text can be combined, for both concern the benefits of infrastructure to women. 3) the last sentence is loose and seems a little bit wordy. Sentence (1) in the target text is characteristic of the English way of thinking, developing ideas from the general to the specific, thus its information casting is different from that of the source text.

\section{B. Example 2 [6]}

What is the nature of the scientific attitude, the attitude of the man or woman who studies and applies physics, biology, chemistry, geology, engineering, medicine or any other sciences?

Analyses:

This is the target text of another short Chinese paragraph. The source text contains only one sentence, which begins an article entitled the Scientific Attitude. It is quite clear to the translator that the purpose of the whole article (omitted here) is to try to answer what the scientific attitude is, so s/he rearranged the order of information: s/he begins the target text with the question "What is the nature of the scientific attitude,...”, thus greatly having adapted the translation to the cognitive perspective of the target readers

\section{CONCLUSIONS}

While the economy is being increasingly integrated and globalized, English has become the international language. Mutual exchanges in political, economic, scientific, diplomatic and other fields across all countries are becoming frequent.

The author of this paper has designed a practical, feasible and easy-to-handle translation mode for scientific discourse, based on information equivalency and information transitivity----two properties of Informatics. This translation mode is designed to cater to the needs of scientific workers who have difficulty comprehending the usually long, multi-structured sentences, which frequently appear in the source scientific texts, and in some ways to accelerate the introduction of western technologies to China and vice versa, so as to contribute to international exchanges and cooperation.

\section{ACKNOWLEDGMENT}

This paper is actually one of the research findings of the project entitled "Translating Metaphors: A Study on C-E Translation Approaches to Conceptually Metaphorical Expressions, Exemplified by Lu Xun's Translated Works” (No.RW201209) of Northwestern Polytechnical University; and is also financially supported by Humanities and Social Sciences foundation of Ministry of Education of China from 2013-2015 ( No. 13xja74002 )

\section{REFERENCES}

[1] Feng Zhi-jie, Feng Gai-ping, Information Equivalency and Transitivity of Target Texts: Basic Binary Standard in Translation [J]. Chinese translators Journal, 1996

[2] Feng Zhi-jie, Chinese-English Translation of Scientific Discourses [M], Beijing: China Translation \& Publishing Corporation, p.210-211, 2000

[3] Feng Zhi-jie, A Guide to C-E Translation for Science [M], Beijing: China Translation \& Publishing Corporation, P. 212-213, 2009

[4] Fu Zu-yun, Basic Theories and Applications of Informatics [M], Beijing: Electronic Industry Press, 2007

[5] Morry Sofer, Translator Self-Training Chinese [M], Rockville: Schreiber Publishing, Maryland, U.S.A, P.23\&37, 2002

[6] Yan Jun-ren, A New Book of Chinese-English Translation of Science \& Technology [M], Beijing: National Defense Industry press, P.99, 2010 